\title{
Anisotropic optical reflection by stepped surfaces
}

\author{
P. L. de Boeij, a) C. M. J. Wijers, and E. Zoethout \\ Faculty of Applied Physics, Twente University, P.O. Box 217, 7500 AE Enschede, The Netherlands
}

(Received 22 January 1996; accepted 20 April 1996)

\begin{abstract}
Discrete dipole calculations of the double cell type have been used to study the anisotropic reflection at normal incidence of stepped $\operatorname{Si}(00 \overline{1}) 2 \times 1$-type surfaces. Only $D_{B}$-type steps have been used. The maximum of the anisotropy turns out to be in a direction rotated with respect to the principal axes, and the anisotropy itself depends strongly on terrace width. Further the crossed polarizer configuration is interesting for experimental work since it has no offset. (C) 1996 American Vacuum Society.
\end{abstract}

\section{INTRODUCTION}

Scanning tunneling microscopy (STM) has given a considerable new impetus to surface science. As a result many detailed features of the surface have become the subject of elaborate studies, one of those features being the step. It extends beyond the scope of this article to give an extensive overview of all research about stepped surfaces mainly because the optical properties of stepped surfaces are the prime interest of this article. There is hardly any literature in this field. The work coming closest is the study by Yasuda and Aspnes ${ }^{1}$ about several Si surfaces. Very recent is the work of Mantese et al. about $\mathrm{Si}(001)$ with high miscut angles. ${ }^{2}$ Further, there exists some theoretical work about the influence of missing row reconstructions on the surface optical response of $\mathrm{Au}$ (Ref. 4) and $\mathrm{Si}^{3}{ }^{3}$ The aim of this article is to study the influence of steps on the optical response of stepped $\mathrm{Si}(00 \overline{1})(2 \times 1)$ surfaces and to understand the extent steps can be ignored when measuring and interpreting the optical anisotropy of this type of surface. This question is relevant since samples used in practice always have some kind of miscut. The steps are well-defined structures on top of a surface. This opens unique possibilities to study the short range interactions by varying the density of the steps. Since steps are by their very nature microscopic structures on the surface, the best way to study them is by means of a microscopic model. Unfortunately, terraces with a width of more than 10 lattice spacings are beyond the capacity of most microscopic methods particularly electronic structure calculation methods. We will show in this article that discrete dipole types of calculations, using the double cell technique, ${ }^{5,6}$ are able to handle such high Miller index surfaces. However the cellular polarizabilities, which are the basic input for this class of calculations, are not yet available from $a b$ initio calculations. The present calculations have been performed using model polarizabilities.

\section{EXTENDED DOUBLE CELL METHOD}

How to calculate the optical response of the surface of a semi-infinite crystalline system using the discrete dipole model has been described by us in a number of articles. ${ }^{5,6}$ The basic idea is that the entire system becomes represented

${ }^{\text {a)} E l e c t r o n i c ~ m a i l: ~ b o e i j @ t n . u t w e n t e . n l ~}$ by a number of discrete sources: the point dipoles $\mathbf{p}_{l}$, located at sites $\mathbf{r}_{l}$. Each dipole becomes linearly induced by the local field $\mathbf{E}_{\mathrm{Loc}, l}$ at the dipole position, according to its polarizability $\alpha_{l}$. In this way, a set of simultaneous linear equations is obtained for any system which must be solved numerically. Hence very large systems and especially semi-infinite systems cannot be treated in a straightforward way. Only if symmetry can be taken into account, being the case for crystalline systems, semi-infinite systems can be transformed to a problem of manageable proportions. The double cell method $^{5,6}$ is one of the most efficient methods to solve the discrete dipole equations for an arbitrary choice of crystalline surfaces.

Two kinds of symmetry are exploited by the double cell method. The translational symmetry remains intact for directions parallel to the surface. Deep inside the crystal, however, the influence of the surface can be ignored, and there effectively, three-dimensional translational symmetry exists. There the response of all bulk dipoles can be efficiently described by normal modes. For this reason, the system is split in the double cell method into a surface and a bulk part, described by two normal modes. Two complex numbers, governing the strength of these normal modes, are the only free parameters for the bulk. All dipole-dipole interactions of the system are taken into account if we consider the interactions between the characteristic dipoles $\mathbf{p}_{i}$ of the surface layer and the bulk normal modes. The characteristic dipole is a single dipole which can represent an entire lattice plane of dipoles as a result of parallel translation symmetry. Upon solution of the dipole-dipole (electrodynamic) interactions governing the system, the individual dipoles of the system are obtained. Now a rather straightforward procedure can be used to obtain the remote optical response (reflection).

To enable treatment of the the optical response of stepped surfaces, the double cell method needs adaptation to handle systems having a triclinic bulk unit cell with two or more dipoles. Until now this method could handle only systems having a simple monoclinic bulk unit cell (with one dipole) ${ }^{5}$ spanned by $\mathbf{s}_{1}, \mathbf{s}_{2}$ and the perpendicular axis $\mathbf{d}_{B}$. If more than one dipole had to be taken into account, we reduced them to effectively one, by means of the commensurability theorem. ${ }^{5}$ Most of the adaptation is shown in Fig. 1 and concerns only the true bulk (normal mode) part of the double 


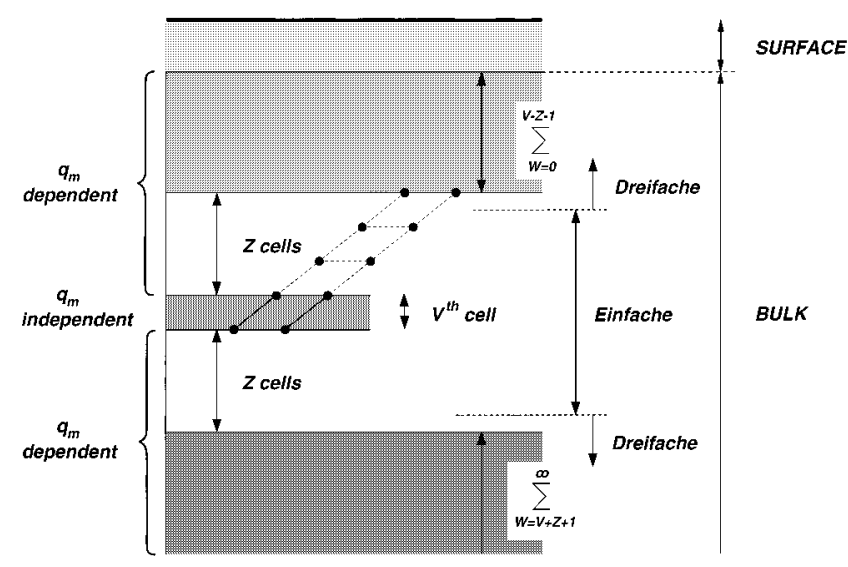

FIG. 1. Schematic view of the double cell geometry with thin triclinic bulk unit cells and the $\pm Z$ layers using the one-fold integral transform.

cell method. The key quantity for this type of discrete dipole calculations is the transfer tensor $\mathbf{f}$, governing the interaction between a dipole and a plane of dipoles. All bulk transfer tensors were evaluated by means of the Ewald threefold integral transform. This integral transform expands the transfer tensor into a series of partial waves controlled by the wave vectors $\mathbf{k}_{p q}$ and transverse projectors $\mathbf{d}_{p q} \cdot{ }^{6}$ With triclinic bulk unit cells the distance between neighboring cells in a direction perpendicular to the surface can become very small, turning down the efficiency of the threefold integral transform. Therefore, for a certain number $Z$, we calculate the interactions due to planes being at most $Z$ bulk unit cells below or above the bulk cell of interest $V$ by means of Ewald's one-fold integral transform ${ }^{7}$ (Fig. 1). This requires a rederivation of the secular matrix/determinant and the interaction equations. Technically the derivation is almost exactly as given in, ${ }^{6}$ but we get a slightly different expression for the secular matrix (determinant):

$$
\overleftrightarrow{\mathscr{H}}_{v w}=\stackrel{\alpha}{\alpha}_{v}^{B-1} \delta_{v w}-\alpha_{o}^{-1} \sum_{z=-Z}^{+Z} \overleftrightarrow{\mathbf{f}}_{u V w(V+z)} \exp \left(i z \mathbf{k}_{m} \mathbf{d}_{B}\right)
$$

$$
\begin{aligned}
& +\alpha_{0}^{-1} \sum_{p q}\left[\frac{\exp \left\{\left[i \mathbf{k}_{p q}\left(\mathbf{r}_{v}^{B}-\mathbf{r}_{w}^{B}+Z \mathbf{d}_{B}\right)-\mathbf{k}_{m} Z \mathbf{d}_{B}\right]\right\}}{1-\exp \left[i\left(\mathbf{k}_{m}-\mathbf{k}_{p q}\right) \mathbf{d}_{B}\right]} \overleftrightarrow{\mathbf{d}}_{p q}\right. \\
& \left.+\frac{\exp \left\{\left[i \mathbf{k}_{p q}\left(\mathbf{r}_{v}^{B}-\mathbf{r}_{w}^{B}-Z \mathbf{d}_{B}\right)+\mathbf{k}_{m} \mathbf{d}\right]\right\}}{1-\exp \left[i\left(\underline{\mathbf{k}}_{p q}-\mathbf{k}_{m}\right) \mathbf{d}_{B}\right]}\right] \overleftrightarrow{\mathbf{d}}_{p q}
\end{aligned}
$$

where we have used the following definition for the normal modes:

$$
\mathbf{p}_{v V}=\sum_{m=1}^{M} \nu_{m} \mathbf{u}_{m v} e^{i V \mathbf{k}_{m} \mathbf{d}_{B}}
$$

where $\mathbf{k}_{m}$ is the wave vector, $\mathbf{u}_{m v}$ the eigenvector, and $\nu_{m}$ the strength of the normal modes. Further, we use for the bulk sites of the dipoles $\mathbf{r}_{v V}$ the definition $\mathbf{r}_{v V}=\mathbf{r}_{v}^{B}+\mathbf{d}_{S}+V \mathbf{d}_{B}$, where $\mathbf{r}_{v}^{B}$ are the sites of the dipoles within the bulk unit cell, and $\mathbf{d}_{S}$ is the vector connecting the origins of bulk and surface unit cell. Equation (1) reduces to the result given in Ref. 6 , by making $Z=0$. The crucial difference is in the first summation. All transfer tensors $f_{v V w}(V+z)$ are calculated now, using the one-fold integral transform. In a similar way, we get the expression for the four different types of matrix elements of the double cell interaction matrix $\mathscr{C l}$ :

$$
\mathscr{C l}_{i j}^{S S}=\stackrel{\leftrightarrow}{\alpha}_{i}^{-1} \delta_{i j}-\alpha_{0}^{-1} \stackrel{\mathbf{f}}{i j}_{i j},
$$

$$
\begin{aligned}
& \mathscr{C}_{n j}^{B S}=-\frac{i k^{2}}{2 \epsilon_{0}\left|\mathbf{s}_{1} \times \mathbf{s}_{2}\right|\left|k_{z}\right|} \exp \left[i \mathbf{k}\left(\mathbf{d}_{s}-\mathbf{r}_{j}\right)\right] \hat{\mathbf{t}}_{n}^{T}, \\
& \mathscr{L}_{n m}^{B B}=-\frac{i k^{2}}{2 \epsilon_{0}\left|\mathbf{s}_{1} \times \mathbf{s}_{2}\right|\left|k_{z}\right|} \hat{\mathbf{t}}_{n}^{T} \mathbf{P}_{m}^{B},
\end{aligned}
$$

where $\mathbf{r}_{i}$ is a surface dipole site, $\hat{t}_{n}$ the direction of the analyzer and $P_{m}^{B}$ is defined as

$$
\mathbf{P}_{m}^{B}=\mathbf{P}_{m}^{B}(\mathbf{k})=\frac{\sum_{w=1}^{N_{B}} \exp \left(-i \mathbf{k} \mathbf{r}_{w}^{B} \mathbf{u}_{m w}\right)}{1-\exp \left(i\left[\mathbf{k}_{m}-\mathbf{k}\right] \mathbf{d}_{B}\right)} .
$$

The superscript labels $S$ and $B$ will always refer to surface and bulk, respectively. The only place where we see explicit changes due to the one-fold integral transform, is for the $S B$ type of matrix elements. We will use frequently the anisotropic azimuth $\Omega$, for this article, the angle between the electric field and $\hat{\mathbf{x}}$. The inhomogeneous vector of the interaction equations remains unchanged. ${ }^{6}$ Equations (1)-(7) comprise the bulk of the modifications necessary to let our software handle oblique bulk unit cells with any number of dipoles.

When more than (effectively) one dipole occupies the bulk unit cell, the calculation of the normal mode parameters slows down substantially. Therefore, the performance of the root searching procedure, used in the normal mode part of our discrete dipole package, has been improved. Historically, a general purpose Broyden routine has been used to find the roots from the modulus of the determinant, but this turned out to be far too slow. In a second version, we used a quadratic (complex) extrapolation of the secular determinant while searching for zeros. In the present version, we have used a method which does not use the value of the determinant as such. In this method, we bring the secular matrix to triangular shape, using full pivoting of the matrix elements. 


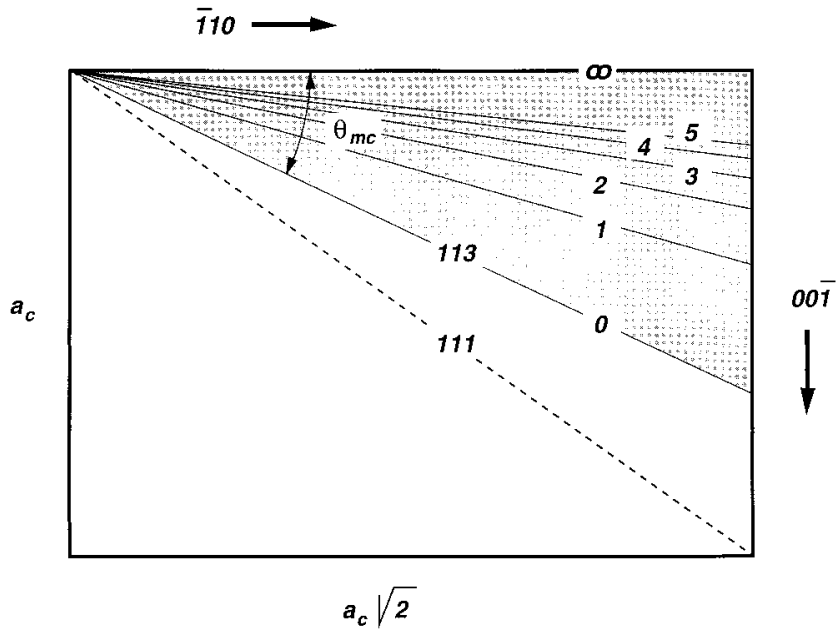

FIG. 2. Intersection of the $\overline{1} 10-00 \overline{1}$ plane and the conventional cube showing miscut angle $\theta_{m c}$ (the integer numbers refer to $N_{D}$ ).

For the triangular secular matrix we know we can write the determinant as:

$$
\|\mathscr{A}\|=\prod_{i=1}^{N_{B}} A_{i i} .
$$

This product will become zero if and only if one of the diagonal elements becomes zero. We are looking for zeros of the diagonal elements: since we have done full pivoting, the zeros will always be found at the lower right corner of the matrix. The point to notice is the matrix element which becomes zero also approaches zero in a linear way even for two-fold degenerate matrices. Using a Regula-Falsi-like technique for the last two diagonal matrix elements, the relevant zeros were found very fast. The advantages of this procedure are clear: It handles matrices of any size, uses linear extrapolation, and creates highly integrated code, since we can use the triangular matrix at the root immediately to produce the normal mode eigenvectors.

\section{STEPPED SURFACES AND GEOMETRY}

The basic geometry of stepped surfaces on $\mathrm{Si}(00 \overline{1})$, has been given by Chadi, ${ }^{8}$ and we follow his treatment and arguments here. We use in this study only double layer stepped surfaces. Of the two types of double layer steps, only the $D_{B}$-type was found to be stable, ${ }^{8,9}$ so we use this type of step to create terraces. Terraces enclosed between $D_{B}$-type steps have all surface dimers oriented in the same direction, parallel to the step edge. For this type of stepped surfaces, the origin of a change in anisotropy can only be in the amount of step edges. If we would have used single step surfaces, there would have been two types of terraces with dimer orientation perpendicular to each other-then the change in anisotropy would depend on both the amount of step edges and the ratio of the surface areas with different orientation.

Chadi gives only isolated steps in his article. A topview of the $D_{B}$-type step is shown in the right panel of Fig. 5. This topview shows the atomic lattice. The atomic lattice as such cannot be used directly for discrete dipole calculations. Ideally, each atom should be represented by one dipole; however, such correspondence does not work. ${ }^{10}$ For semiconductors, the discrete dipole model overestimates the short range interactions. Partially this effect can be overcome by assigning one dipole to each couple of atoms, as Mochán and Barrera did. In this study, we do the same, but this forces us to devise a suitable dipole lattice. The dipole geometry-used by us-is shown in Figs. 3 and 4. The dipole site containing the rebonded atom is, to a certain extent, questionable. From the position of the rebonded atom itself, we can choose the middle of either of two downward directed bonds, both choices being arbitrary. The reconstruction of the $\mathrm{Si}(00 \overline{1})$ $2 \times 1$ surface is commonly called rows of dimers reconstruction, those rows being perpendicular to the $D_{B}$ steps. In addition, we use lines of dimers, being all dimers on the same line parallel to the steps. Rows of dimers belonging to successive terraces have to shift over a $a / 4$ distance parallel to the step edge. On the next terrace, this shift can be the same

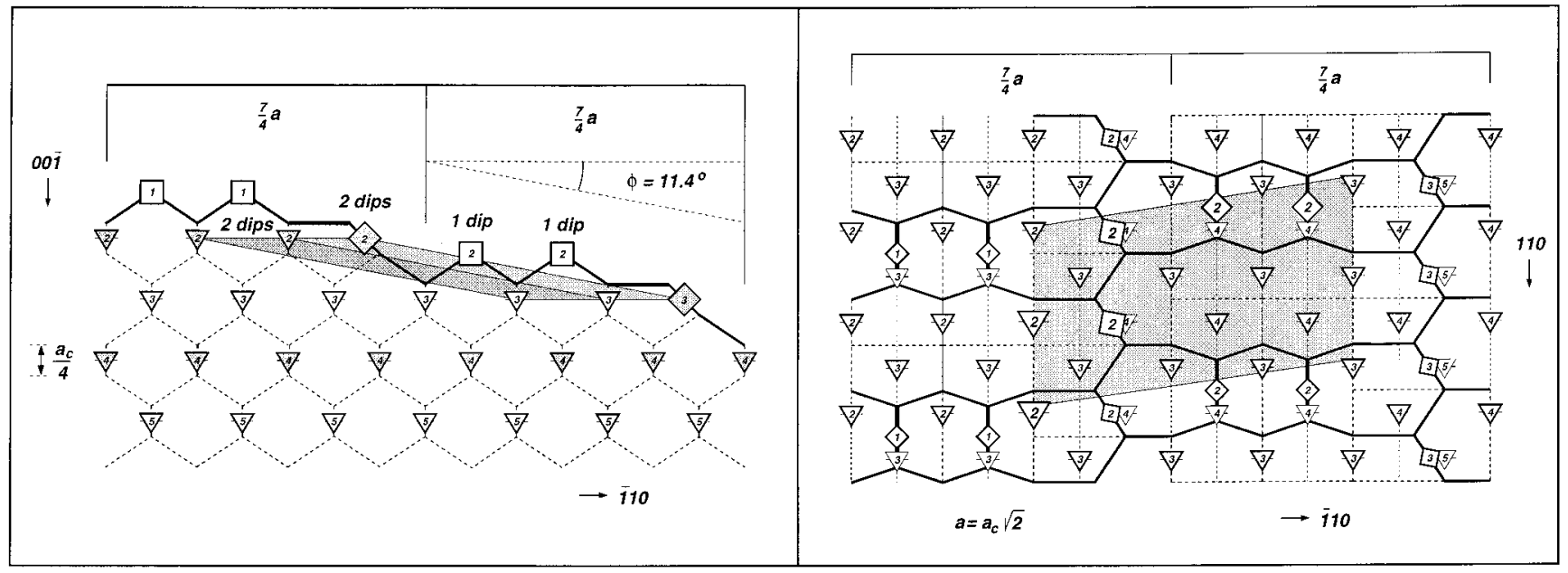

Fig. 3. Location of dipole sites used in the calculations. Left: sideview $\mathrm{Si}(00 \overline{1}), 2$ dimer lines seen from (110). Right: topview $\mathrm{Si}(00 \overline{1}), 2$ dimer lines seen from (001). 


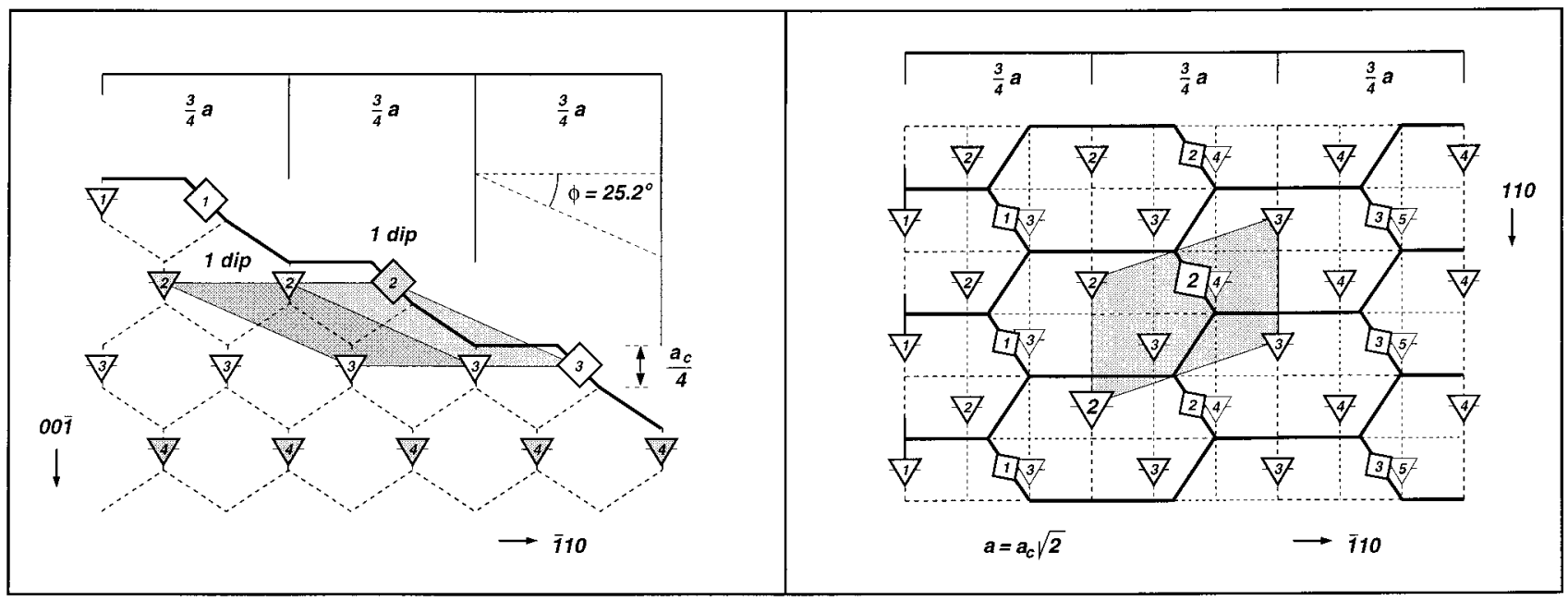

FIG. 4. Location of dipole sites used in the calculations. Left: sideview Si (113) seen from (110). Right: topview Si (113) seen from (001).

or reversed, resulting in two different types of surfaces. We chose a continued shift, yielding an oblique surface unit cell.

We use $D_{B}$-type stepped surfaces, having the miscut angle $\theta_{m c}$ in the (110) (001)-plane of the original $2 \times 1$ reconstructed $(00 \overline{1})$-surface, as shown in Fig. 2. Only planes in the shaded area are considered. The limiting cases are the (001)and $(11 \overline{3})$-surface-the latter plane having only $D_{B}$-type steps and no dimers. The terraces themselves were supposed to have the $(00 \overline{1}) 2 \times 1$ reconstruction. The type of surface morphology obtained in this way is valid for orientations as steep as (117). Beyond that point, nonrebonded step edges are introduced and surface periodicity becomes less well defined [at least until (114), $\left.\theta_{m c}=19.5^{\circ}\right]^{11}$ So our modeling of terraces, having less than 2 dimer lines of width, is not very realistic, but we have added them, nevertheless, to get a better feeling for possible trends.

To obtain a stepped surface having a terrace width containing $N_{D}$ dimer lines requires a miscut angle $\theta_{m c}$ given by

$$
\tan \left(\theta_{m c}\right)=\left(\frac{\sqrt{2}}{3+2 N_{D}}\right) .
$$

For the cases studied here, we list values of $\theta_{m c}$ in Table I.

The coordinates of the sites will be given first in a base frame spanned by the (110), $(\overline{1} 10),(00 \overline{1})$ directions. For all surfaces with $N_{D} \neq 0$, there are two dipoles in the bulk unit cell and $2+N_{D}$ dipoles in the free surface unit cell. The two

TABLE I. $\theta_{m c}$ as a function of \# of dimer lines $N_{D}$.

\begin{tabular}{cc}
\hline \hline$N_{D}$ & Miscut angle $\theta_{m c}\left({ }^{\circ}\right)$ \\
\hline 0 & 25.239402 \\
1 & 15.793169 \\
2 & 11.421754 \\
3 & 8.930142 \\
4 & 7.326037 \\
5 & 6.208545 \\
$\infty$ & 0.000000 \\
$(111)$ & 35.26439 \\
\hline \hline
\end{tabular}

bulk dipoles are located at $(0,0,0)$ and at $a\left(\frac{1}{2}, 0,0\right)$ with respect to the origin of the bulk unit cell, where $a=\sqrt{2} a_{c}, a_{c}$ being the conventional lattice parameter (5.43 $\AA$ for $\mathrm{Si})$. Those sites will not be affected by a rotation to the surface oriented frame. The two dipoles marking the step have sites $\boldsymbol{\tau}_{S}^{1,2}$, given in the base frame by

$$
\begin{aligned}
& \boldsymbol{\tau}_{S}^{1}=a\left[0, \frac{1}{2}\left(1-\frac{1}{8} \sqrt{2}\right), 0\right], \\
& \boldsymbol{\tau}_{S}^{2}=a\left[\frac{1}{2}, \frac{1}{2}\left(1-\frac{1}{8} \sqrt{2}\right), 0\right] .
\end{aligned}
$$

The $N_{D}$ terrace dipoles have sites $\tau_{S}^{i}$ given in the base frame by

$$
\boldsymbol{\tau}_{S}^{2+i}=a\left[\frac{1}{8}, 1+\frac{1}{2}(i-1), \frac{1}{16} \sqrt{2}\right] .
$$

Those sites are with respect to the origin of the free surface unit cell. The origins of bulk and free surface unit cell are connected by $\Delta \boldsymbol{\tau}=-a \hat{\mathbf{y}}$ and the third vector $\mathbf{d}_{B}$ spanning the bulk unit cell is in the base frame $-a \hat{\mathbf{y}}$. From these data, the proper coordinates in the (stepped) surface oriented frame are obtained by a coordinate transformation over $\theta_{m c}$, as described. In the surface frame the parallel translational symmetry is governed by the spanning vectors $\mathbf{s}_{1}$ and $\mathbf{s}_{2}$ :

$$
\begin{aligned}
& \mathbf{s}_{1}=a(1,0,0), \\
& \mathbf{s}_{2}=a\left[\frac{1}{4}, \frac{1}{4} \sqrt{2+\left(3+2 N_{D}\right)^{2}}, 0\right] .
\end{aligned}
$$

This description of the geometry does not include the two limiting cases $(00 \overline{1})$ and $(11 \overline{3})$. The first should be obvious and the $(11 \overline{3})$ case follows basically the treatment given here but is halved in the (110) direction.

The last part of the description of the configuration used concerns the polarizabilities. All polarizabilities will be derived from bulk optical data using Clausius-Mossotti in the following way:

$$
\left(\frac{\alpha}{\alpha_{0}}\right)=\left(\frac{a_{c}}{a}\right)^{3} \frac{3}{16 \pi}\left(\frac{\epsilon-1}{\epsilon+2}\right),
$$




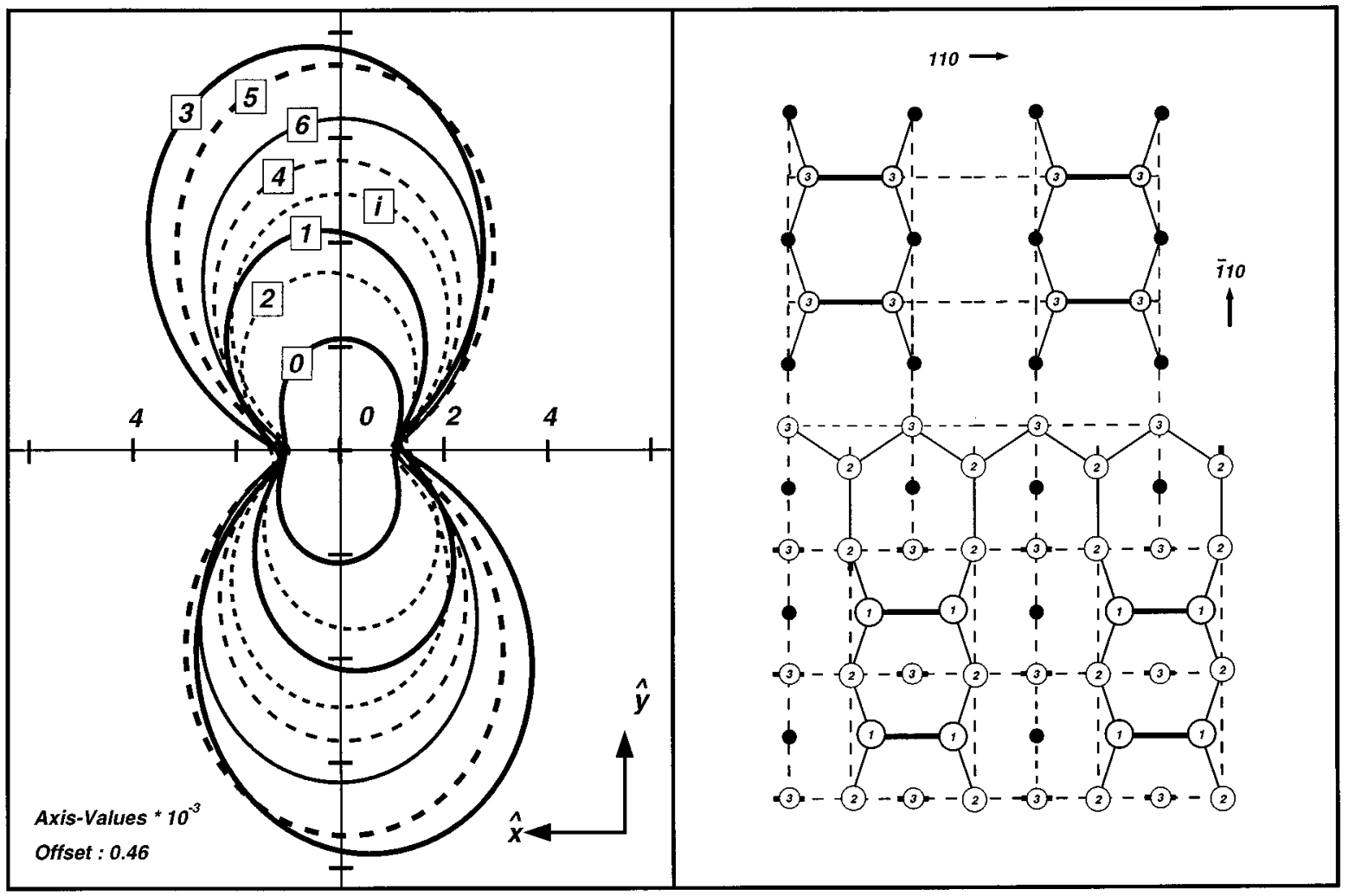

FIG. 5. Left: Azimuthal plot pp reflectance as a function of $\Omega$. Right: topview separate $D_{B}$ step (atomic sites).

where $\epsilon$ is the relative dielectric constant, $a_{c} / a$ depends on the surface $\left[(11 \overline{3})\right.$ is different], and $\alpha_{0}=4 \pi \epsilon_{0} a^{3}$. The numerical value for $\alpha_{0}$ equals $5.0398573 \times 10^{-38} \mathrm{~F} \mathrm{~m}^{2}$ for all cases except for (113). There the value is $6.299821 \times 10^{-39} \mathrm{~F} \mathrm{~m}^{2}$. Calculations are isotropic if only isotropic polarizabilities are used and show the structural contribution to the anisotropy. To investigate a possible electronic structure influence, anisotropic polarizabilities are required. We have restricted this anisotropy to the surface dipoles, the edge and terrace ones. Taking the $z$ direction parallel to the dimer axis, we introduce anisotropy as

$$
\begin{aligned}
& r_{\text {Ani }}=\alpha_{z z} / \alpha_{x x, y y}, \\
& \alpha_{y y}=\alpha_{x x} .
\end{aligned}
$$

We have kept in our calculations this intrinsic anisotropy modest, effectively only $2 \%$. Hence, we use only $r_{\text {Ani }}=1.0$ (isotropic), and $r_{\mathrm{Ani}}=1.02$ (anisotropic). The anisotropic polarizability has to be rotated to its proper position. This requires interchange of indices for the terrace dipoles. The edge dipoles have been rotated twice by means of an Euler rotation. First they have been given a rotation over the Euler angles $\Psi=-45^{\circ}, \theta=-45^{\circ}$ to get the correct values for the base frame: ${ }^{12}$

$$
\alpha_{p q}^{L}\left(\theta_{M}\right)=\sum_{i, j} S(\phi, \theta, \Psi)_{p i} \alpha_{i j}^{M}\left[S(\phi, \theta, \Psi)^{-1}\right]_{j q},
$$

where $S(\phi, \theta, \Psi)$ is the Euler transformation matrix as given by Goldstein. ${ }^{13}$ The second rotation from the base frame to the surface frame is over the angle $\theta_{m c}$ and is applied to the entire system. After that, the polarizability tensors no longer contain zero valued components. Numerical values for the anisotropic components of the polarizability prior to rotation are $\alpha_{z z}=1.914052 \times 10^{-2}+i 2.085819 \times 10^{-4}$ and $\alpha_{x x, y y}$ $=1.876522 \times 10^{-2}+i 2.044921 \times 10^{-4}$ in units of $\alpha_{0}$ [except (113)].

\section{RESULTS}

Using the previously mentioned geometry and optical input data, model calculations have been performed for perpendicular incidence. We have used $p$ type of polarization for the incoming light, letting the direction of polarization coincide with anisotropic azimuth $\Omega$. The frequency for all calculations has been $\hbar \omega=3.0 \mathrm{eV}$ unless stated otherwise. The polarizabilities were also calculated at this frequency. The first results of this type ( $p p$ combinations) are shown in Fig. 5, left panel. For those calculations, all surface dipoles have been given anisotropic polarizabilities. The numbers shown in boxes in the curves themselves indicate the number of dimer lines in the reconstruction. The two limiting cases are given by the label (i) for $(00 \overline{1})$, and 0 for $(11 \overline{3})$. Since the anisotropy is weak as compared to the average signal, an offset of 0.46 has been subtracted to emphasize the results. The right panel shows the atomic configuration of a single $D_{B}$ step, shown in the same orientation as the left panel. It is 


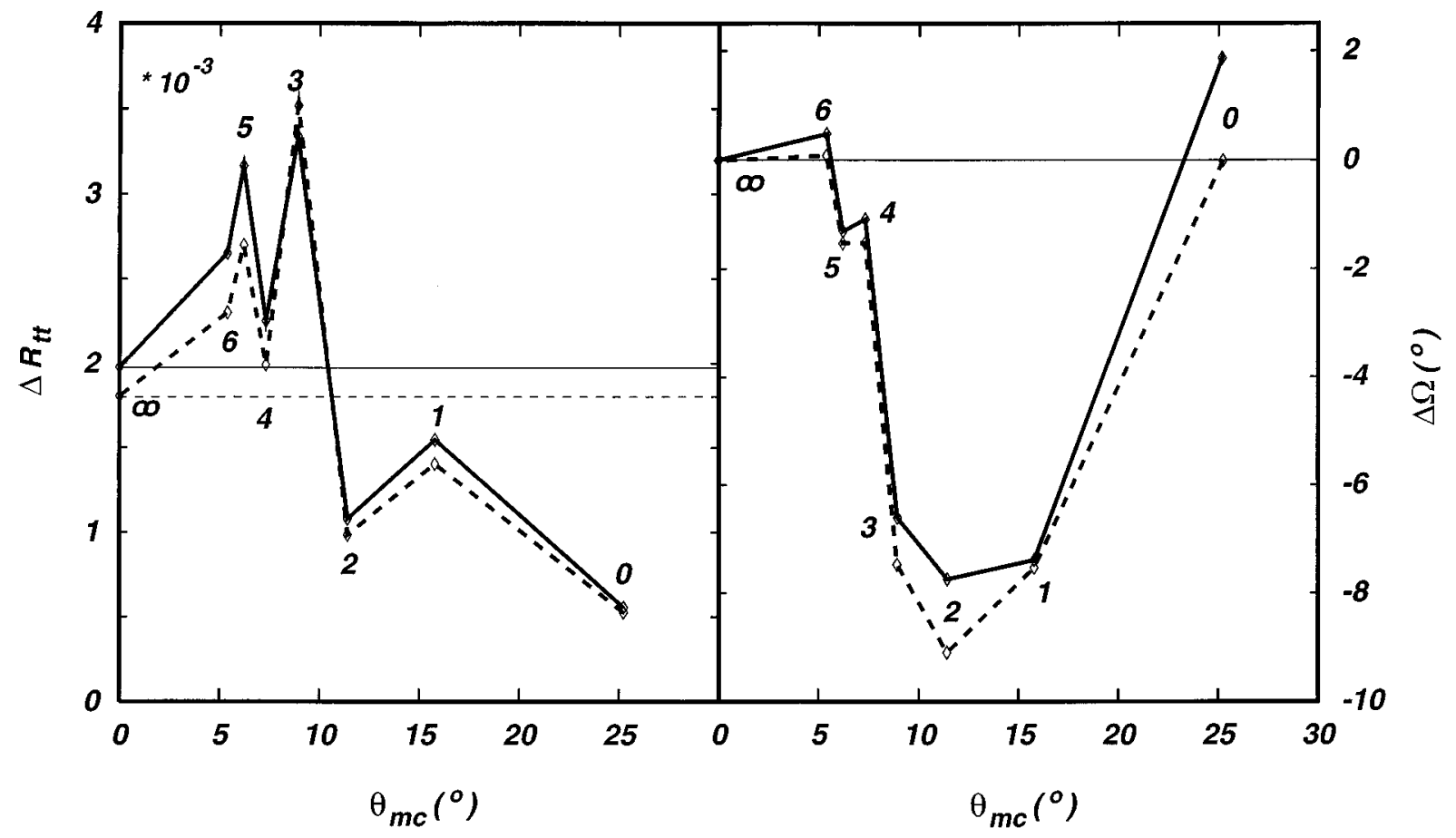

FIG. 6. Left: Top-top modulation of $R_{\mathrm{pp}}$ (reference anisotropies indicated by horizontal lines). Right: anisotropic azimuth offset $\Delta \Omega$. Solid line: anisotropic results. Dashed line: isotropic results.

immediately clear that the $p p$ plots are not exactly lattice oriented. Neither the bulk lattice, nor the step seems to control the orientation of the plots.

The maximum value of the $p p$ reflectance is obtained for the polarization direction approximately perpendicular to the steps. We know from experience that single linear chains of dipoles have increased emission when the polarization direction is parallel to the chains. If we assume the dimer rows will act like separate chains of dipoles, we can expect that for these stepped surfaces, the maximum reflectance is obtained for polarization parallel to the rows. We will use the anisotropy of the original $(00 \overline{1})$ surface as a reference in the remainder of this article. It is surprising to see that this reference anisotropy is not the maximum anisotropy. By intuition, we would expect the interruption of the dimer rows by the steps to decrease the reference anisotropy of the unstepped surface. Apparently this is not the case. Why the steps sometimes happen to increase, or to decrease the reference anisotropy has no simple explanation. It is clear, nevertheless, that steps have a strong influence on this anisotropy. They can increase or decrease it by a factor of two. Finally, we notice that the plots are tilted into the same direction as the shift of the dimer rows, belonging to consequent terraces. This is also expected.

The maximum terrace width shown here is for $N_{D}=6$. Technically we can go well beyond $N_{D}=10$, but at the expense of highly increased runtimes. The reason for the increment is the number of dipoles required by the matching layer between the free surface cell and the bulk normal mode regime. This number increases from 60 to 90 , going from $N_{D}=1$ to $N_{D}=6$. Even those large numbers are not enough to keep the convergence rate at the level observed for the $(00 \overline{1})$ and $(11 \overline{3})$ limiting cases. We have always used such a number of matching dipoles that convergence had no influence on our results.

To have more direct access to the relevant parameters, we have made a fit through the calculated data. We have used as a fit function:

$$
R_{p p}(\Omega)=A+B \cos \left(N_{\Omega} \Omega\right)+C \sin \left(N_{\Omega} \Omega\right),
$$

where we have taken $N_{\Omega}=2$. After performing the least squares procedure, we arrived at a very good fit. The fit parameters have subsequently been transformed to more directly understandable data as follows:

$$
\begin{aligned}
& R_{p p}(\Omega)=A+\Delta R_{t t} \sin \left[N_{\Omega}(\Omega-\Delta \Omega)\right], \\
& \Delta R_{t t}=\sqrt{B^{2}+C^{2}}, \\
& \Delta \Omega=\arctan (B / C) / N_{\Omega} .
\end{aligned}
$$

We will focus upon the top-top modulation $\Delta R_{t t}$ and the azimuthal shift $\Delta \Omega$. The solid curves in Fig. 6 belong to the anisotropic cases, shown as azimuthal plots in the left panel of Fig. 5. From the top-top modulation, we learn that the anisotropy is not a smooth function of the miscut angle or terrace width. There is something like an even-odd behavior superimposed on top of a simple curve having a single maximum. This maximum shows up for about 3 dimer lines of terrace width. It turns out that for $p p$ reflectance the top-top modulation cannot be used experimentally as an indicator for the terrace width. The same holds for the azimuthal shift. The influence of the azimuthal shift $\Delta \Omega$ has been explicitly 
omitted by Yasuda and Aspnes in their article. ${ }^{1}$ The only misoriented (001) surface in their paper has a $\theta_{m c}=4^{\circ}$ corresponding to a terrace width of 9 dimer lines, just beyond the region where we find the strongest shifts. This justifies their assumptions. The minimum of the azimuthal shift is at $N_{D}=2$ and these shifts arrive faster at the reference level $(\approx 0)$ for increasing terrace width, than the corresponding top-top modulation.

To figure out how much of the effects found is structural (geometry, dipole sites), and how much is electronic (polarizability), we have repeated the calculations with isotropic polarizabilities throughout. Those isotropic results are also shown in Fig. 6 as the dashed curves. Although the results clearly change, the overall pattern remains unaffected. Only for the azimuthal shift $\Delta \Omega$, we see that for the $(11 \overline{3})$ case the tilt disappears. This also happens for terraces with $N_{D} \geqslant 6$, but there the shift is already very low. For the values of $r_{\text {Ani }}$ used in this article (about 1.02), the anisotropy of stepped surfaces is predominantly structural. For a larger value of $r_{\text {Ani }}$ stronger electronic contributions will be expected. From these calculations we get a first impression how a larger $r_{\text {Ani }}$ will affect the anisotropy. Better polarizability data have to be obtained from other calculations.

Since most of the observed behavior has to be understood as structural, we have also tried to figure out whether wiggling plays a role. Those rapid oscillations of the dipole strength have been observed by us before ${ }^{5}$ and we associate them with structural effects. The new phenomenon, which might play a role here is lateral wiggling, wiggling along the surface. Previous observations of wiggling were always perpendicular to the surface. The even-odd behavior of the

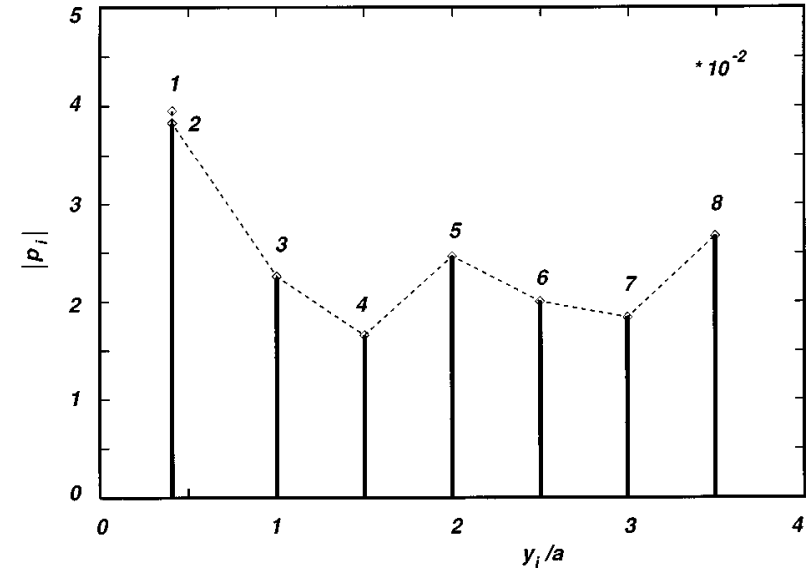

FIG. 7. Modulus of dipole strength $|\mathbf{p}|_{i}$ as a function of $y .|\mathbf{p}|_{i}$-axis values to be multiplied by $10^{-2}$

top-top modulation might be caused by it. Since wiggling depends strongly on frequency, we have repeated the anisotropic calculations with $\hbar \omega=6.0 \mathrm{eV}$. The results indicate that the influence of wiggling is weak. A more direct observation is given by the individual dipole strengths (in Fig. 7). All dipole strengths of the free surface layer are shown as a function of the $y$-coordinate. Indeed lateral wiggling with a two-fold period can be observed.

Discrete dipole calculations depend critically on the choice of the dipole geometry. To verify how sensitive our present calculations are as to this point, we have repeated the calculations with the edge dipole moved to the position of

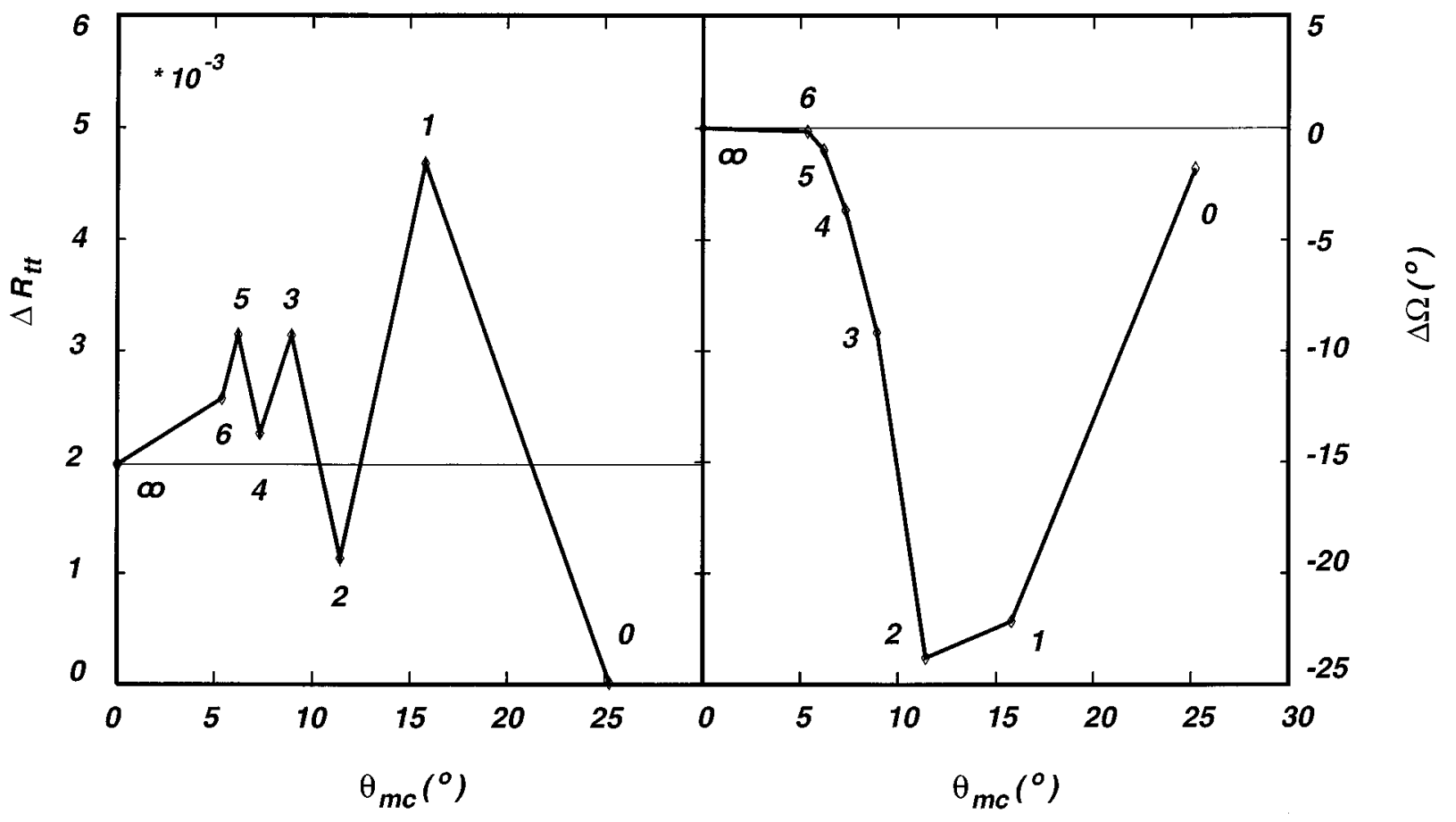

FIG. 8. Left: top-top modulation of $R_{\mathrm{pp}}$ (horizontal line: reference). Right: anisotropic azimuth offset $\Delta \Omega$. Anisotropic results, $\hbar \omega=3.0$ eV edge dipole at rebonded atom position. 


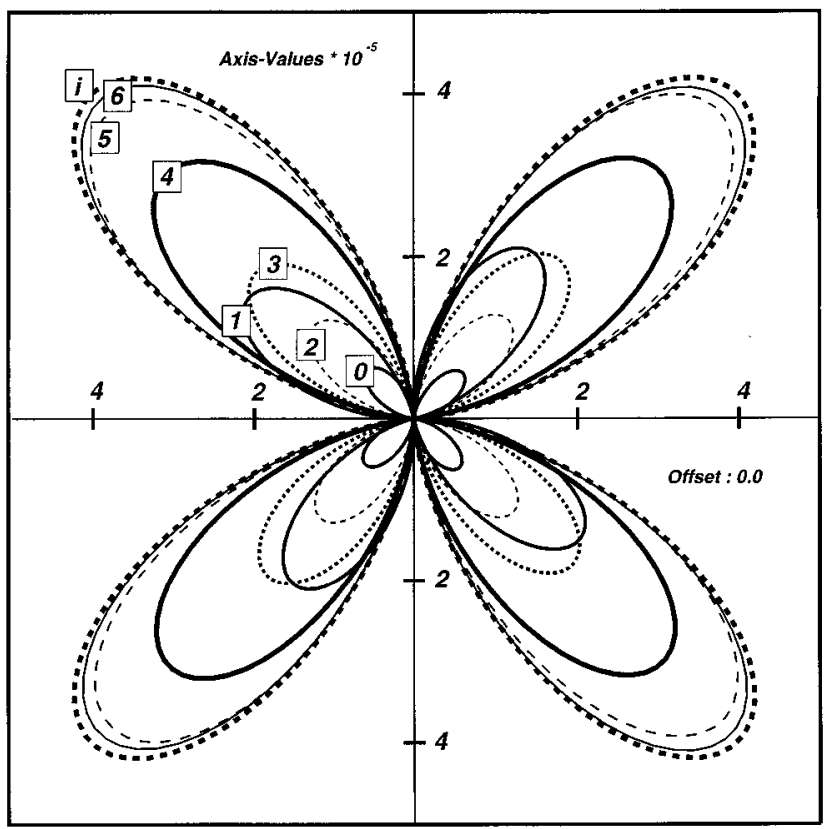

FIG. 9. Azimuthal plot ps reflectance as a function of $\Omega$.

the rebonded atom. To remove asymmetry due to the tilting of the polarizability tensor of this site, we have given it the same polarizability tensor as the dimers. The results are shown in Fig. 8. There are a few major differences. The top-top modulation for the $N_{D}=1$-case has increased by a factor of 3 , the anisotropy for the $N_{D}=0$-case has virtually disappeared, and the azimuthal shifts have increased system- atically by a factor of 2 . Those changes are large, but the overall behavior has not been affected by the shift of the edge dipole. When $N_{D} \geqslant 3$, the changes are not significant.

For the stepped surfaces treated here, another outspoken new feature of the calculation is that the polarization no longer remains in the plane of incoming polarization. For this reason, we have investigated also this ps-type (crossed polarizer) configuration. The azimuthal results for the anisotropic case are shown in Fig. 9. The result is a series of four-lobed patterns. Those patterns are obtained without using an offset. At first glance, those patterns look fundamentally different from the ones shown in Fig. 5, but this is only seemingly. The major point is that there is really no offset. The complex ps-reflection coefficient has the same two period behavior as the pp component, but since it is symmetric around zero, the modulus-procedure $R=r^{*} r$ turns it into a four period phenomenon.

The ps-type of reflectances have again been fitted by us by means of Eq. (16), using now $N_{\Omega}=4$. The results obtained this way, are shown in Fig. 10. Again the solid lines represent the anisotropic cases. A major result shows up already after inspection of the top-top modulation. For $N_{D}>2$ this modulation is a monotonous function of $N_{D}$. The even-odd behavior is absent. As compared to their pp-counterparts, the modulation has dropped by two orders of magnitude. Further the $N_{D}=\infty$ [original (001)] results turn out to be the highest now. Only in the results for the azimuthal shift, still the even-odd behavior is visible but not as pronounced as for the pp cases. The differences between the azimuthal shift data comparing pp and the ps-cases are smaller than between the corresponding top-top modulation data. We have also

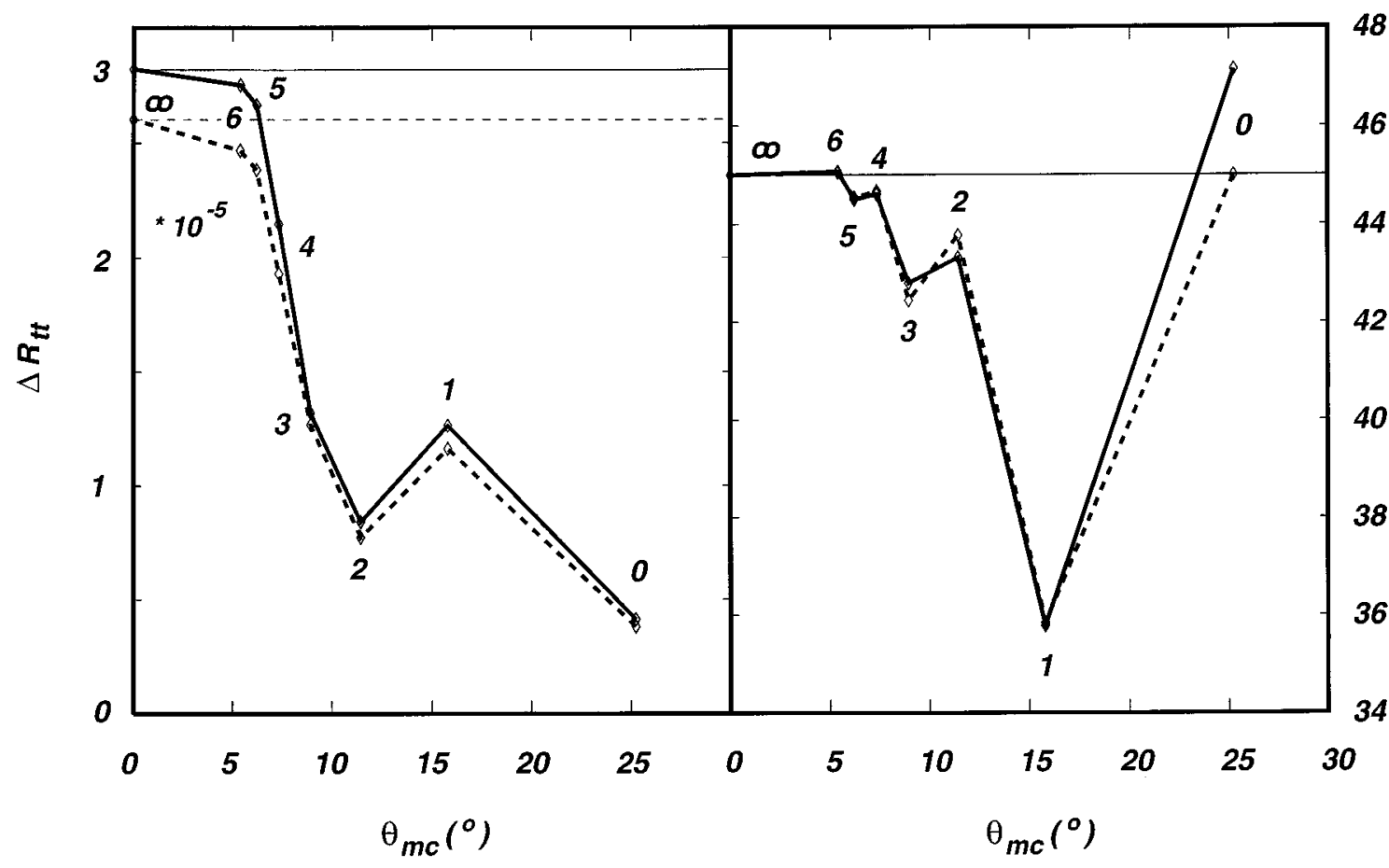

FIG. 10. Left: top-top modulation of $R_{\mathrm{ps}}$ (horizontal lines: reference). Right: anisotropic azimuth offset $\Delta \Omega$. Solid line: anisotropic results. Dashed line: isotropic results. 
repeated the calculations using isotropic polarizabilities. Again the influence is small.

The major conclusion from the ps type of reflection coefficients is in the way experiments can be done. Despite the fact that the ps-type results are in strength two orders of magnitude below the pp-type results, those data can be obtained without offset. This means that the full sensitivity of the detection system can be used. If our calculations turn out to be reliable, it means that the ps results can be easier correlated with the terrace width, since the ps-response curve is monotonic.

\section{CONCLUSION}

In this article, we have shown a number of model calculations based on the discrete dipole model for the optical response of stepped surfaces. We have shown that it is possible to perform those calculations for terrace widths, having up to 10 dimer lines. Most of the step influence is gone for terraces being more than 6 dimer lines wide. Measurements on $\mathrm{Si}(00 \overline{1})$, with up to $4^{\circ}$ miscut, need no correction for those effects. For higher miscut angles, the influence of steps becomes too strong to be ignored. The step contribution to the anisotropy can be as large as the anisotropy of the original Si $(00 \overline{1}) 2 \times 1$ surface. That is the regime where interesting fundamental work can be done. The point where our calculations and the experimental data will possibly deviate will give an indication for the effective range of the electrodynamic interaction. This asks for further experimental work into this direction. For perpendicular incidence, as studied here, the main axes of the polarization plots do not follow the crystallography of the surfaces. From our calculations, follows that the easier way to measure the influence of steps on optics is by measuring in crossed polarizer (ps) mode. The expected advantages are in a better sensitivity and in easier interpretation of results.

${ }^{1}$ T. Yasuda, D. E. Aspnes, D. R. Lee, C. H. Bjorkman, and G. Lucovsky, J. Vac. Sci. Technol. A 12, 1152, (1994).

${ }^{2}$ L. Mantese, U. Rossow, and D. E. Aspnes, Proceedings of the ACSI-3 Conference, 1995 (to be published).

${ }^{3}$ G. P. M. Poppe, H. Wormeester, A. Molenbroek, C. M. J. Wijers, and A. van Silfhout, Phys. Rev. B 43, 12122 (1991).

${ }^{4}$ S. Wang, W. L. Mochán, and R. G. Barrera, Phys. Rev. B 42, 9155 (1990).

${ }^{5}$ G. P. M. Poppe and C. M. J. Wijers, Physica B 167, 221 (1990).

${ }^{6}$ C. M. J. Wijers and G. P. M. Poppe, Phys. Rev. B 46, 7605 (1992)

${ }^{7}$ G. P. M. Poppe, C. M. J. Wijers, and A. van Silfhout, Phys. Rev. B 44, 7917 (1991).

${ }^{8}$ D. J. Chadi, Phys. Rev. Lett. 59, 1691 (1987).

${ }^{9}$ H. J. W. Zandvliet (private communication).

${ }^{10}$ W. L. Mochán and R. G. Barrera, Phys. Rev. Lett. 55, 1192 (1985).

${ }^{11}$ A. A. Baski, Naval Research Laboratory, Washington DC (to be published).

${ }^{12}$ R. Bersohn, Y. Pao, and H. L. Frish, J. Chem. Phys. 45, 3184 (1966).

${ }^{13}$ H. Goldstein, Classical Mechanics (Addison-Wesley, Reading, MA 1974). 